\title{
Introduction to Minitrack: Multi-criteria Decision Analysis and Support Systems
}

\author{
Amita Goyal Chin \\ Virginia Commonwealth Univ \\ agchin@vcu.edu
}

\author{
H. Roland Weistroffer \\ Virginia Commonwealth Univ \\ hrweistr@vcu.edu
}

\author{
Rakesh Sarin \\ UCLA \\ rakesh.sarin@anderson.ucla.edu
}

\begin{abstract}
Almost all decisions people make are based on multiple factors or criteria. Decision makers generally pursue multiple, and often conflicting, objectives. A feasible solution that is optimum with respect to all such objectives or decision criteria almost never exists, and a satisfactory compromise solution is generally sought. Multi-criteria decision analysis as a field of research deals with problem theory and solution approaches directly involving multiple decision criteria. This minitrack focuses on solution approaches, technology, and systems that support decision-making under consideration of multiple decision criteria.
\end{abstract}

\section{Introduction}

Multiple criteria decision problems generally do not possess a mathematically well-defined optimum solution. Thus the best the decision maker (DM) can aspire is to find a satisfactory compromise solution from among the efficient (non-dominated) solutions. Usually there is no known and explicit utility function representing the preferences of the DM, and interactive solution techniques are often proposed to identify the preferred solution or perhaps a manageable set of desirable compromise solutions.

Multi-criteria decision analysis (MCDA), an active area of research since the 1970s, deals with structuring and solving decision problems with explicit consideration of multiple decision criteria. Thousands of articles and dozens of books have been devoted to this field, with several regular conferences and conference tracks focusing on this area.

This minitrack on MCDA is in its eighth year as part of the HICSS program, and has attracted an average of ten paper submissions each year, with an average acceptance of five submitted papers. In addition, at HICSS-45, Jyrki Wallenius from Aalto University in Helsinki, Finland, gave a keynote address on Multi-objective Optimization - Different Interaction Styles and an Approach as part of this minitrack, and at HICSS-47, Raimo Hämäläinen, also from Aalto University, moderated a panel on The Process of Multicriteria Decision Support-Practical Approaches, New Perspectives, and Behavioral Issues in this minitrack.

\section{Papers in this minitrack}

This year, two papers have been accepted for this minitrack that cover two important topics in multicriteria decision analysis - crowdfunding and value focused thinking.

Jun Chen, Long Chen, Chen Qian, and Ke-fan $\mathrm{Xie}$ in their paper Decision making in crowdfunding under risk analysis, examine an emerging international, internet-based economic activity, crowdfunding, and the potential risks that influence the decision-making process of the investors that partake in such financial transactions. Specifically, the authors quantitatively analyze the risk factors associated with crowdfunding as faced by potential investors and present analytical decision-making models. Furthermore, they develop an evolutionary game simulation system to gauge the dynamic decision-making process.

Kweku-Muata Osei-Bryson in the paper $A n$ Integrated Framework for The Value Focused Thinking Methodology, presents an integrated framework for the Value Focused Thinking (VFT) methodology that attempts to improve the elicitation and definition of domain objectives. Specifically, the framework addresses decision context, types of relationships between objectives, quality of the description of the objectives, and the need to create value-based alternatives. 